\title{
Bericht vom Operationskurs "Nahttechniken in der Urologie“" am 30. und 31.8.1991 in Mainz
}

\author{
K. Lang
}

Urologische Klinik und Poliklinik im Klinikum der Johannes Gutenberg-Universität Mainz

In anderen operativen Fachrichtungen sind Kurse zur Vermittlung von Nahttechniken seit längerem eingeführt, in der Urologie jedoch bisher noch nicht. Am 30. und 31.8.1991 fand unter der Leitung von Prof. Dr.med. $R$. Hohenfellner in der Urologischen Klinik und Poliklinik der Johannes-Gutenberg-Universität Mainz der erste Operationskurs "Nahttechniken in der Urologie“ statt. 34 Teilnehmer, auch aus dem deutschsprachigen Ausland, nahmen an diesem Kurs teil.

Zur Durchführung der Nahtübungen, die Mittelpunkt des Kurses waren, standen formolkonservierte Schweinepräparate zur Verfügung, an denen ein realitätsnahes Operieren möglich war. Die Darmmukosa war rosa eingefärbt, was die operativen Übungen erleichterte. Die einzelnen Operationen wurden durch ein kurzes Video vorgestellt, in dem die entscheidenden Schritte am Schweinepräparat demonstriert wurden, ergänzt durch intraoperative Vergleichsaufnahmen vom Originalsitus. Parallel zu den Übungen der Teilnehmer wurde von Mitarbeitern der Urologischen Klinik die jeweilige Operation live am Schweinepräparat über Großbildprojektion demonstriert, wobei immer wieder auf spezielle Abläufe hingewiesen wurde. Je zwei Teilnehmer operierten gemeinsam.

Inhaltlich wurde der Kurs mit einem Vortrag von Dr. K. Felkl (Fa. B. Braun-Dexon) über Nahtmaterialien eingeleitet. Als erste Operation wurde eine Antirefluxplastik nach Gregoir, danach ein Psoas-Hitch durchgeführt. Nach einer Harnleiter-End/End-Anastomose, bei der die einreihige Einzelknopfnaht von Muskularis und umgebendem Gewebe unter Aussparung der Mukosa geübt wurde, und einer aortorenalen Gefäßanastomose (fortlaufende Allschichtnaht, spezieller Einsatz von Haltenähten zur Verhinderung des versehentlichen Fassens der Gefäßrückwand) endete der erste Tag des Kurses.

Der zweite Tag war schwerpunktmäßig den Nahttechniken an Dick- und Dünndarm gewidmet. Es wurde mit einer End/End-Dünndarmanastomose und einer Ileoascendostomie die seromuskuläre Naht in Einzelknopf- und fortlaufender Technik vorgestellt und durchgeführt. Anhand der Bildung eines Ileozökalpouches (MainzPouch) wurde die fortlaufende überwendliche Allschichtnaht zur Bildung des Pouches demonstriert, ebenso wie

Akt. Urol. 23 (1992) 91

(c) Georg Thieme Verlag Stuttgart · New York die notwendigen Schritte zur Bildung eines kontinenten Stomas: die Mesopräparation, die Vorbereitung der Staplerklammernaht (Teilentfernung von Klammern zur Verhinderung von freiliegenden Anteilen der Klammernaht Steinbildung) sowie die Invagination und Nippelfixation. Die Harnleiter-Darmimplantation am Pouch wurde in „Open End“-Technik durchgeführt, die Technik nach Goodwin/Hohenfellner wurde im Video demonstriert.

Eine weitere Möglichkeit der Bildung eines kontinenten Stomas unter Verwendung der Appendix wurde per Video vorgestellt, der Vortrag von Dr. Bürger über die Grundlagen der Kontinenz beim Appendixstoma ergänzte das Video.

Die neue Variante der Ureterosigmoidostomie, der „Sigma-Rektum Pouch/Mainz-Pouch II“, bei dem Rektum und Sigma antimesenterial miteinander anastomosiert und am Promontorium fixiert werden, fand lebhaftes Interesse.

Der Kurs wurde durch den Vortrag von Prof. Hohenfellner über „trouble shooting“ beim MainzPouch beschlossen.

Den Veranstaltern des Nahtkurses, dessen Organisation in der Hand von PD Dr.med. S. C. Müller lag, ist es nach übereinstimmender Meinung der Teilnehmer gelungen, das selbstgesteckte Ziel zu erreichen, nämlich die Vermittlung klassischer und neuerer Nahttechniken in der Urologie. So jedenfalls muß man die Auswertung der anonymen Fragebogen deuten, die am Ende der Veranstaltung ausgegeben wurden und um eine Benotung der Punkte: Organisation, Verpflegung, Anleitung, Betreuung, Präparate, Skripts, Material und Rahmenprogramm zwischen 1 (sehr gut) und 6 (ungenügend) baten. Das arithmetische Mittel der Noten war in allen Kategorien besser als 2, bis auf den Punkt Verpflegung. In ihm offenbarten sich die lukullischen Ansprüche der Teilnehmer, denen die Veranstaltung leider nicht gerecht werden konnte. Abgesehen davon, läßt sich als Fazit wohl sagen, daß dieser Kurs $z u$ einer ständigen Einrichtung in der deutschsprachigen Urologie werden sollte.

\section{K. Lang}

Urologische Klinik und Poliklinik im Klinikum der

Johannes-Gutenberg-Universität

Langenbeckstr. 1

6500 Mainz 